\title{
Large Deflection Electrostatic Spiral Actuator with Twisted-beams
}

\author{
Sachiya Okamoto Student Member (University of Hyogo, okamoto.sachiya@elnics.eng.himeji-tech.ac.jp) \\ Nobuaki Shimamoto Non-member (University of Hyogo) \\ Yuko Matsushita Non-member (University of Hyogo) \\ Takayuki Fujita Member (University of Hyogo, fujita@eng.u-hyogo.ac.jp) \\ Kazusuke Maenaka Member (University of Hyogo, maenaka@eng.u-hyogo.ac.jp) \\ Yoichiro Takayama Non-member (University of Hyogo, takayama@eng.u-hyogo.ac.jp)
}

Keywords : polysilicon surface micromachining, twisted-beam, electrostatic, spiral actuator, large deflection, MEMS

Many electrostatic actuators fabricated by the technology of micro electro mechanical systems (MEMS) have been reported. Especially, the stress-induced or bimetal-like actuators have merits of the simple structure and fabrication steps with relatively large stroke. The stress-induced actuators have a internal tensile-stress layer on a base material, for example a cantilever beam with a $\mathrm{Cr}$ layer on Poly-Si layer. The cantilever beam is curved from the substrate by its the internal stress.

Conventional stress-induced spiral-actuators are not suitable for increasing a total stroke. In this section, we present a novel spiral actuator whose stroke is a sum of each stroke of constituent beams. We introduce "twisted-beam" as shown in Fig. 1. This beam simultaneously has a curvature to out-of-plane (same as conventional stress-induced actuators) and torsional deflection around longitudinal direction of the beam. The torsional deflection makes the end of curved beams to a horizontal direction, and the next beam connected to that end will start at horizontal plane.

For the convenience of following discussion, we use the word "curved-angle" to represent the angle between the longitudinal median line and the substrate at free end of the beam and "twisted-angle" for the rotational angle between the end face of the beam to the substrate (See Fig. 1(a)). The twisted-beam is formed by many basic cells as shown in Fig. 1(b). The cell of the twisted-beam consists of a relatively wide stress-induced region (base material with stress layer) and a non-stressed region (only base material). In the stress-induced region, three dimensional deflection along a spherical surface occurs, whereas there is no deflection in the non-stress region. The connection between the

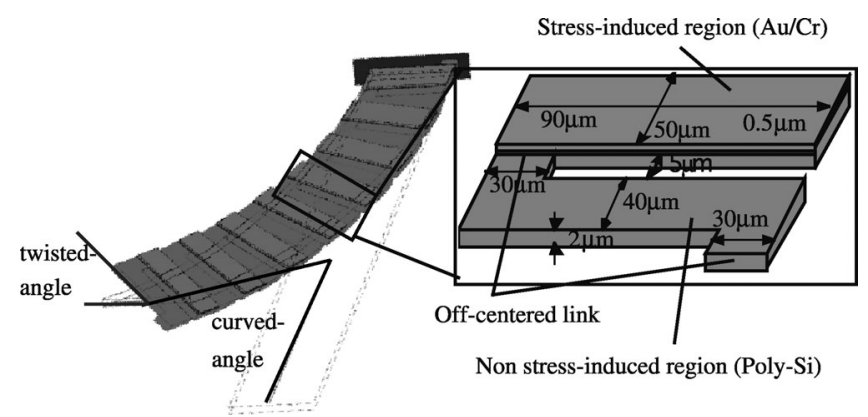

(a)Twisted-beam $\quad$ (b)A cell of twisted-beam

Fig. 1. Structure of twisted-beam and its cell stress-induced region and the non-stressed region is off-centered and the non-stressed region is inclined to the stress-induced region. Thus the cell has not only a curved-angle but twisted-angle. The curved-angle mainly depends on the length of the stress layer, and the twisted-angle depends on the width of the stress layer and the offset of the connection. The appropriate design is required for the optimized cell structure where the twisted-angle compensates the curved-angle so that next beam starts at horizontal plane. The dimensions of Fig. 1 were determined so that the twisted-angle becomes equal with the curved-angle for the compensation of the effect of the curvature to the out-of-plane.

Figure 2 shows SEM photographs of the spiral actuators with twisted-beams (a) and conventional stress-induced beams (b). Clearly, large deflection can be seen in the actuator with twisted-beams. On the contrary, the actuator with conventional beams not only has small deflection but also becomes entangled. The detailed discussion is omitted here, but this phenomenon, the torsional deviation in x-y plane, can be theoretically calculated for the actuators with relatively large stress in the stress-induced region. Maximum displacement to $\mathrm{z}$-axis is about $750 \mu \mathrm{m}$ whereas the displacement or shift in $x-y$ plane is around $100-300 \mu \mathrm{m}$. This characteristic must be taken into account for the system design including a load of the actuators.

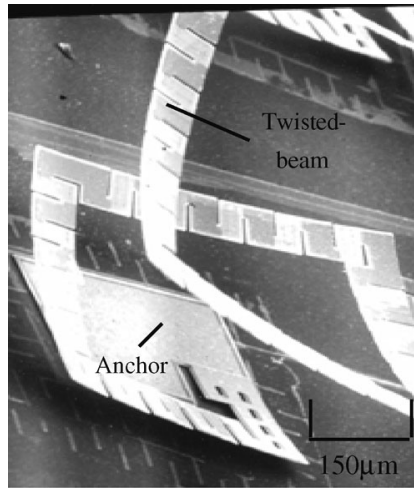

(a) With twisted-beams

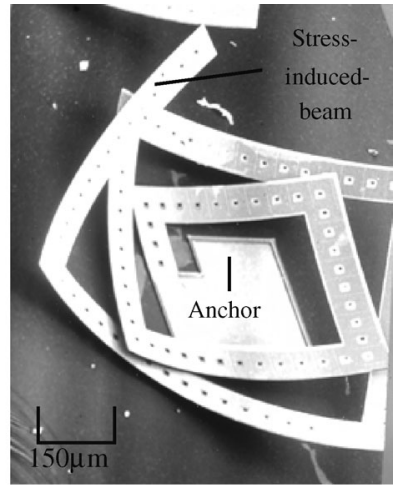

(b) Straight beams with different number of cells
Fig. 2. SEM photographs of fabricated electrostatic spiral actuator 


\section{Large Deflection Electrostatic Spiral Actuator with Twisted-beams}

$\begin{array}{ll}\text { Sachiya Okamoto* } & \text { Student Member } \\ \text { Nobuaki Shimamoto* } & \text { Non-member } \\ \text { Yuko Matsushita* }^{*} & \text { Non-member } \\ \text { Takayuki Fujita* }^{*} & \text { Member } \\ \text { Kazusuke Maenaka* }^{*} & \text { Member } \\ \text { Yoichiro Takayama* } & \text { Non-member }\end{array}$

In this paper, we propose a large-deflection electrostatic spiral-actuator with novel twisted-beams fabricated by surface micromachining. The stress-induced or bimetal-like actuators have merits of simple structure and fabrication steps with relatively large stroke. The novel stress-induced actuator presented here is composed of "twisted-beams", which have not only out-of-plane curvature but also rotation along its longitudinal direction. When the twisted-beams are used as components of a spiral actuator, the total stroke of the actuator becomes a sum of each deflection of the beams, and the stroke is much larger than conventional stressinduced spiral actuators. The experimental results of the spiral actuator show the maximum deflection of about $750 \mu \mathrm{m}$, which is extremely large for stress-induced-actuators.

Keywords : polysilicon surface micromachining, twisted-beam, electrostatic, spiral actuator, large deflection, MEMS

\section{Introduction}

Many electrostatic actuators fabricated by the technology of micro electro mechanical systems (MEMS) have been reported. Especially, the stress-induced or bimetal-like actuators have merits of the simple structure and fabrication steps with relatively large stroke. The stress-induced actuators have a internal tensile-stress layer on a base material, for example a cantilever beam with a $\mathrm{Cr}$ layer on Poly-Si layer. The cantilever beam is curved from the substrate by its the internal stress. The cantilever can be attracted to the substrate by electrostatic force when the voltage is applied to the beam and the substrate through the insulating layer on the substrate. Many types of actuators using this mechanism have been reported ${ }^{(1)-(4)}$, which include RF-switches ${ }^{(5)(6)}$, micro valves ${ }^{(7)}$, micro turbines ${ }^{(8)}$, optical switches ${ }^{(9)}$, etc. Some of these devices are already in the market. For increasing area factor and decreasing operation voltage, the actuators with spiral or conical shape have also been reported ${ }^{(10)(11)}$. In such devices, operation voltage and area factor can be reduced, however they cannot increase the actuation stroke because the stroke of the beam is defined only by the distance between the free end and fixed point of the spiral but not by total length of the beam in the spiral. Thus, for increasing total stroke, the novel concept has to be introduced.

In this report, we propose the "twisted-beam" which has both curvatures to out-of-plane and rotation along longitudinal direction of the beam, and examine the spiral actuators constructed with the twisted-beams.

\section{Operation of Conventional Stressinduced Cantilevers}

Fig. 1 shows the structure of a conventional stress-induced

\footnotetext{
* Department of Electrical Engineering and Computer Sciences, Graduate School of Engineering, University of Hyogo 2167 Shosha, Himeji, Hyogo 671-2201, Japan
}

cantilever beam, which has a $\mathrm{Au} / \mathrm{Cr}$ layer for a tensile-stress layer on Poly-Si as a base material. Because the base material is uniformly covered with the stress layer, the curvature of the film is constant over the whole surface. Therefore, the beam lies on the circular arc depending on the film thickness, Young's modulus and internal stress. The radius of curvature $R$ of the stress-induced surface is given by ${ }^{(9)}$,

$$
\begin{aligned}
& R=\frac{E_{2} h\left[3 m+K\left\{n(1+n)^{2}\right\}^{-1}\right]}{6\left(m \sigma_{2}-\sigma_{1}\right)} \ldots \ldots \ldots . . . . . \\
& K=1+4 m n+6 m n^{2}+4 m n^{3}+m^{2} n^{2} \\
& m=\frac{E_{1}}{E_{2}}, n=\frac{h_{1}}{h_{2}}, h=h_{1}+h_{2}
\end{aligned}
$$

where $E$ is the Young's modulus, $h$ is the thickness, $\sigma$ is the residual stresses. The subscripts 1 and 2 indicate the values for $\mathrm{Au} / \mathrm{Cr}$ and Poly-Si, respectively. The out-of-plane displacement at the free end of the beam $z$ is described by

$$
z=R\left(1-\cos \left(\frac{L}{R}\right)\right)
$$

where $L$ is the length of the cantilever beam. This result implies that the maximum displacement of the spiral structure is defined only by the radius of the spiral and is the same as the straight cantilever with the equal length to the radius of the spiral structure. In the following, the above discussion will be verified using FEM analysis.

Fig. 2 shows the result of the FEM analysis for a spiral actuator, which has three straight-beams connected in series at right angle. The beam 1, whose one end is fixed to the substrate, is curved with the radius described by eq. (1). The free end of the beam 1 has an angle from the substrate, $\theta$, due to the curvature of the beam. Thus, the beam 2 , which is perpendicularly connected to the 


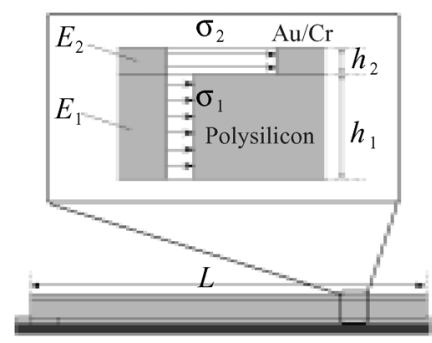

(a) Before releasing the sacrificial layer

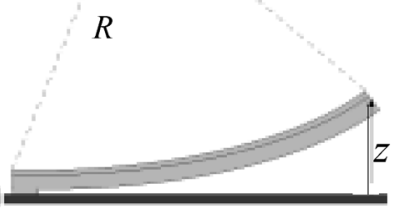

(b) After releasing the sacrificial layer
Fig. 1. Schematic of conventional stress-induced cantilever beam

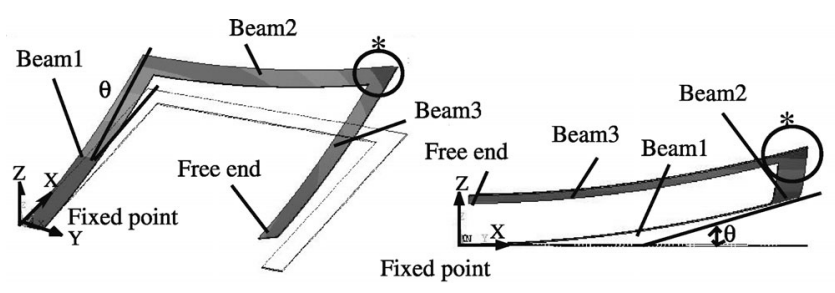

(a) Perspective view

(b) Lateral view

Fig. 2. Result of FEM analysis for conventional spiral actuator

end of the beam 1, has an initial inclined angle at its starting point. Accordingly, the beam 3 will start to curve with an angle toward the substrate (see* point in Fig. 2). Thus, the z-position at the free end of the beam 3 is lowerd from its starting point in this dimension and total stroke does not equal to a sum of each deflection of the beams.

\section{Twisted-Beams}

3.1 Concept As described above, conventional stressinduced spiral-actuators are not suitable for increasing a total stroke. In this section, we present a novel spiral actuator whose stroke is a sum of each stroke of constituent beams. We introduce "twisted-beam" as shown in Fig. 3. The twisted-beam is formed by some of cells which contain a stress-induced region and non-stressed region. The stressed-induced region and non-stressed region are connected together by off-centered link as shown in Figs. 3 and 4. In the stress-induced region, the beam curves along a spherical surface. The longitudinal curvature makes a vertical displacement of the beam. On the other hand, the transversal curvature in the stress-induced region gives a torsional displacement of the beam because the next cells are connected by off-centered link. Thus the beam simultaneously has a curvature to out-of-plane (same as conventional stress-induced actuators) and torsional deflection around longitudinal direction of the beam. The torsional deflection makes the end of curved beams to a horizontal direction, and the next beam connected to that end will start at horizontal plane. In other words, the end of the beam 2 marked with $*$ in Fig. 2 is rotated clockwise when the beam 2 is twisted-beam. Thus, by using twisted-beams, total stroke of the spiral actuator becomes to be a sum of each stroke of the beams.

3.2 Basic Design For optimization of the device, the relation between a curvature to out-of-plane and torsional deflection is important. For the convenience of following discussion, we use the word "curved-angle" to represent the angle between the longitudinal median line and the substrate at free end of the beam and "twisted-angle" for the rotational angle between

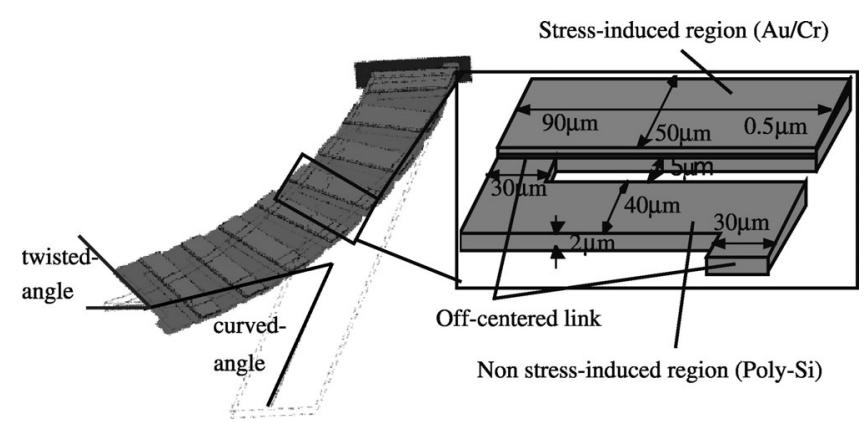

(a) Twisted-beam

(b) A cell of twisted-beam

Fig. 3. Structure of twisted-beam and its cell

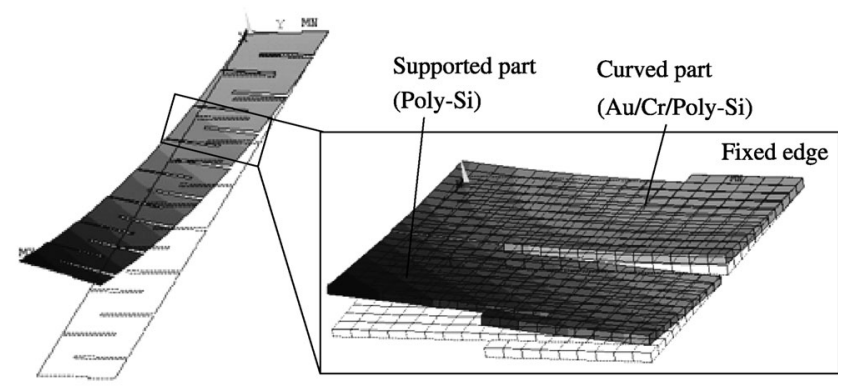

(a) Perspective view

(b) Lateral view

Fig. 4. FEM analysis result of twisted-beam

the end face of the beam to the substrate (See Fig. 3(a)). The twisted-beam is formed by many basic cells as shown in Fig. 3(b). The cell of the twisted-beam consists of a relatively wide stress-induced region (base material with stress layer) and a non-stressed region (only base material). In the stress-induced region, three dimensional deflection along a spherical surface occurs according to eq. (1) (3), whereas there is no deflection in the non-stress region. The connection between the stress-induced region and the non-stressed region is off-centered and the non-stressed region is inclined to the stress-induced region. Thus the cell has not only a curved-angle but twisted-angle. The curved-angle mainly depends on the length of the stress layer, and the twisted-angle depends on the width of the stress layer and the offset of the connection. The appropriate design is required for the optimized cell structure where the twisted-angle compensates the curved-angle so that next beam starts at horizontal plane. The dimensions of Fig. 3 were determined so that the twisted-angle becomes equal with the curved-angle for the compensation of the effect of the curvature to the out-of-plane. Fig. 4 shows the results of FEM analysis of the twisted-beam. The influence of the gravity to deflection of the beam can be neglected (less than $1 \%$ of total deflection) because the weight of the beam is sufficiently small against stiffness of the beam. Fig. 5 shows the twisted-angle and curved-angle for the number of connected cells, where the angles are almost equal regardless of the number of the cells.

The curved-angle directly determines the stroke or maximum deflection of the actuator. In our device, the stress-induced region is a part of the beam and the stroke may be lower than the one for conventional devices in which the stress-induced region covers whole surface of the beam. Fig. 6 indicates the maximum deflections of our device and conventional device as a function of the beam length. The stroke of our device is about $3 / 5$ of conventional one.

3.3 Spiral Actuator Fig. 7 shows an example of the 


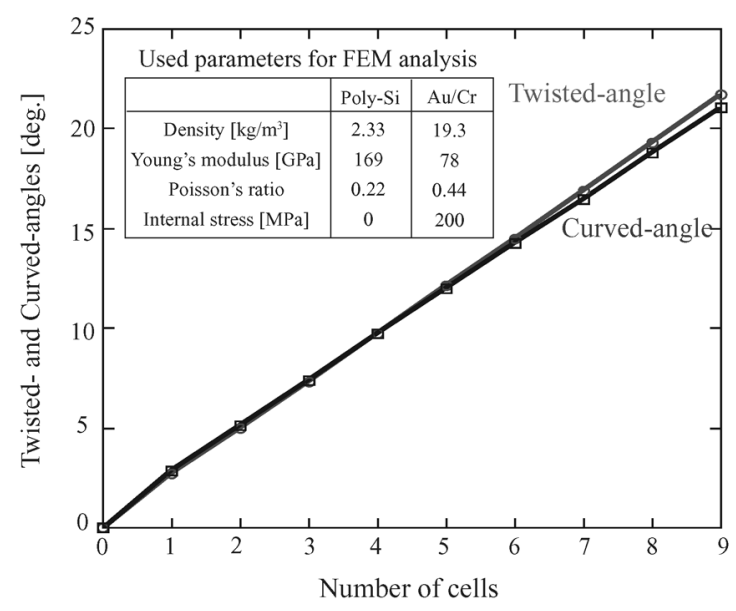

Fig. 5. Curved- and twisted-angles for the number of straightly-connected twisted-cells

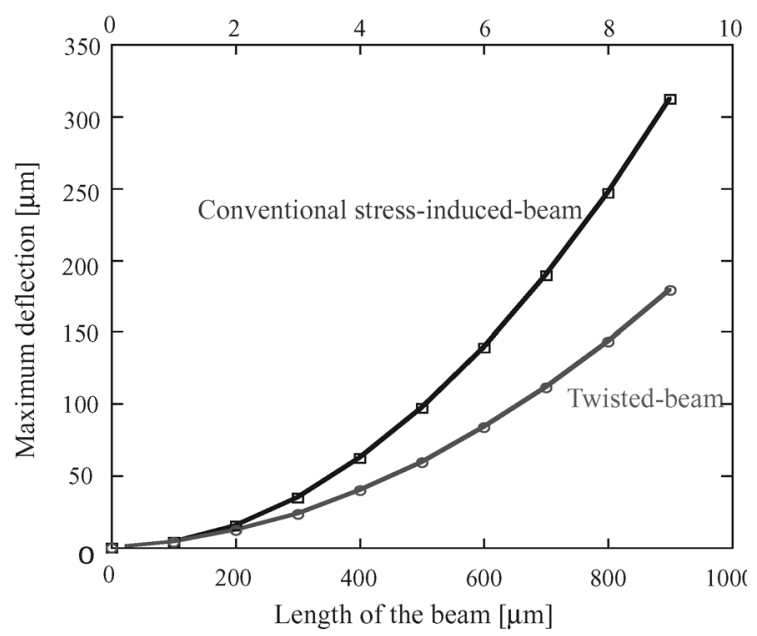

Fig. 6. Maximum deflections of the straight beam

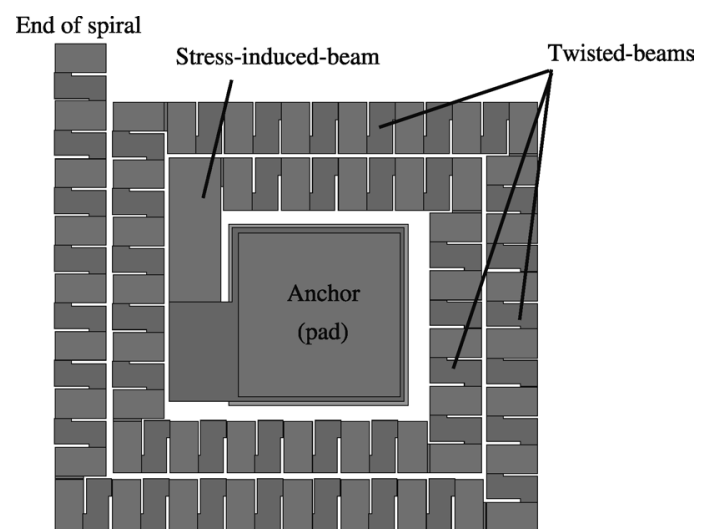

Fig. 7. Schematic view of the spiral actuator with twisted-beams

designed spiral actuator with twisted-beams. A $2 \frac{1}{4}$-turn spiral is anchored to the insulating layer on the substrate at its center. The first or anchored straight-beam is not a twisted-beam but conventional beam. The device size is $850 \times 850 \mu \mathrm{m}^{2}$. Total of 44 cells are used. Fig. 8 shows the results of FEM analysis on the assumption of the internal stress of $200 \mathrm{MPa}$ which is calculated from a combination of $\mathrm{Au} / \mathrm{Cr}$ on Poly-Si films for the stress-induced layer. From horizontal view in Fig. 8, it is clear that

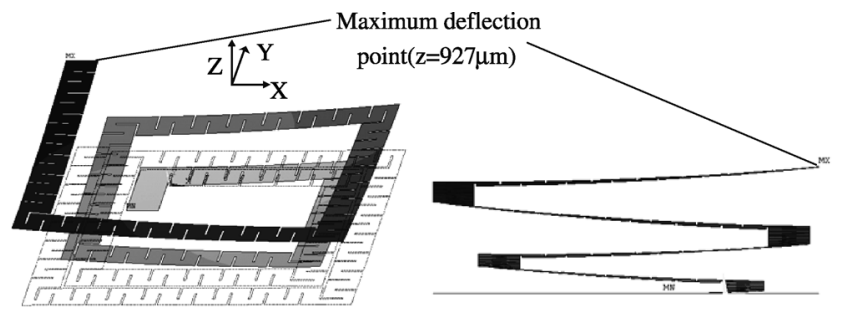

(a) Perspective view

(b) Lateral view

Fig. 8. Results of FEM analysis for spiral actuator with twisted-beams

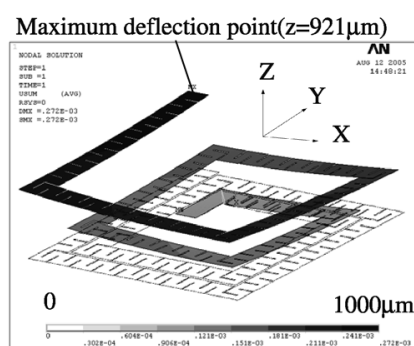

(a) z-axis deflection contour

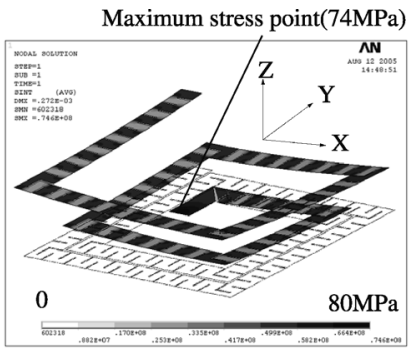

(b) Stress distribution contour
Fig. 9. Stress analysis versus z-axis acceleration (1G)

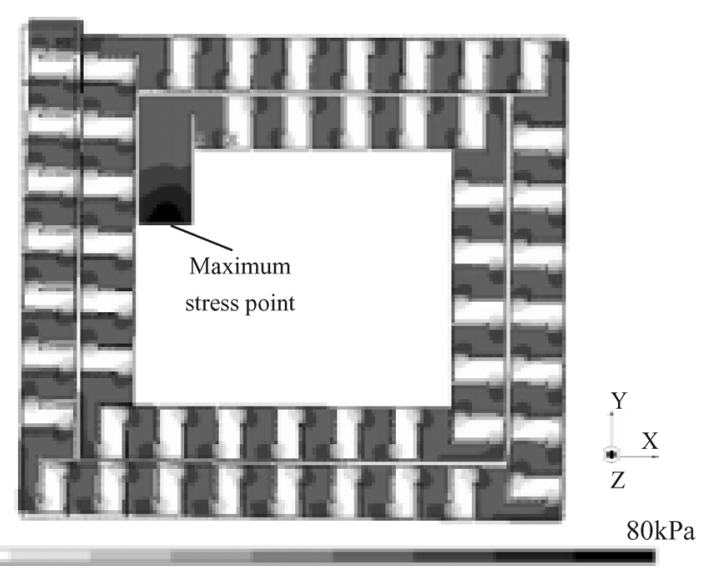

(a) $\mathrm{x}$-axis $(1 \mathrm{G})$

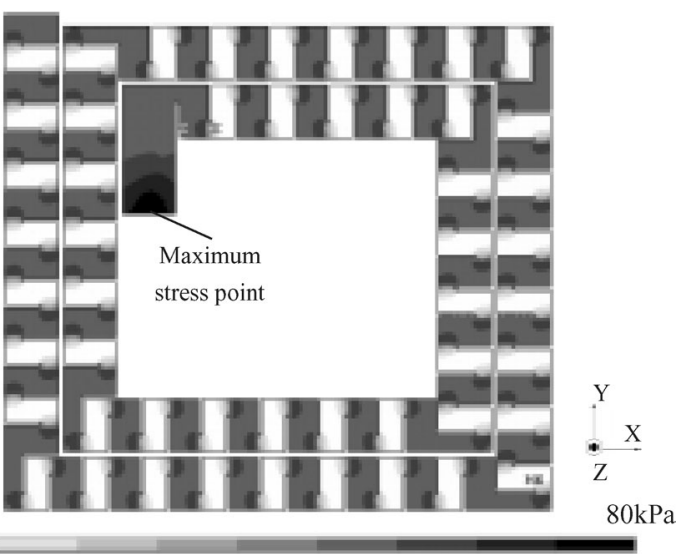

(b) $y$-axis (1G)

Fig. 10. Stress distribution versus horizontal acceleration (Top view) 
the total stroke is a sum of each stroke of the twisted-beams. Maximum displacement or stroke was calculated to be about 930 $\mu \mathrm{m}$ which is extremely large value in micro actuators. As for generation force, the pull up force or restoring force of the spiral spring is calculated to be $29.4 \mathrm{nN}$, whereas the pull down force to the substrate is depend on the applied voltage.

\subsection{Mechanical Strength Against Acceleration or Shock}

Our actuator has an irregular shape in the beams, which is a combination of zigzag pattern. It may bring about stress concentration, and the mechanical strength of the beam should be estimated. Here, we applied the static acceleration to the device, and maximum stress and deviation of the for structure were calculated by FEM analysis. As a load of the actuators, the mass with size of $800 \times 800 \mu \mathrm{m}^{2}$ in a Poly-Si layer was appended to the top of the actuator in the calculation. This simulates an optical mirror driven by the top of this actuator. Fig. 9 shows the deviation and stress distribution when $1 \mathrm{G}$ acceleration is applied to the z-axis. The deviation at free end of the actuator is $-6 \mu \mathrm{m}$, and maximum stress of $74 \mathrm{MPa}$ appears near the anchor point. In Fig. 10, the deviation and stress distribution for $1 \mathrm{G}$ acceleration applied to $\mathrm{x}$ and $\mathrm{y}$ axes are shown. Maximum deviation and stress are less than $10 \mathrm{~nm}$ and $74 \mathrm{kPa}$, respectively. Since the failure strength of the Poly-Si is around 2000-2700 MPa, and our actuator can be proof against an acceleration or shock of several tens of $\mathrm{G}$.

\section{Fabrication Process}

The fabrication process is based on the two-layer polysilicon surface-micromachining process. A schematic view of the process flow is shown in Fig. 11. At first, (a) a silicon wafer is prepared. (b) A $0.36 \mu \mathrm{m}$ thermal oxide is grown on the Si substrate followed by a $0.5 \mu \mathrm{m}$ LPCVD silicon nitride layer as an electrical isolation layer. The deposition temperature and growth pressure of silicon nitride are $830^{\circ} \mathrm{C}$ and 0.5 Torr, respectively. The source gases are mixture of ammonia (100\%) and mono-silane $\left(\mathrm{N}_{2}\right.$ Base $\left.20.0 \%\right)$ with the ratio of $3: 1$. The deposition rate was about $3 \mathrm{~nm} / \mathrm{min}$. (c) Then, a $0.5 \mu \mathrm{m}$ LPCVD polysilicon layer (Poly0) is deposited. The deposition conditions are the temperature of $650{ }^{\circ} \mathrm{C}$ and the pressure of 0.5 Torr. The source gas is mono-silane $\left(\mathrm{N}_{2}\right.$ Base $20.0 \%$ ). The deposition rate was about $8 \mathrm{~nm} / \mathrm{min}$. The wafer is then lithographically patterned with the mask of Poly0 and etched by the polysilicon wet etchant. The polysilicon etchant is the mixture of $\mathrm{HNO}_{3}: \mathrm{HF}: \mathrm{H}_{2} \mathrm{O}(25: 1: 25)^{(12)}$. This solution has high selectivity to a photoresist and a nitride layer. (d) Next, the SOG is coated for planarization. After planarization, the wafer is annealed at $1100^{\circ} \mathrm{C}$ for $90 \mathrm{~min}$ in $\mathrm{N}_{2}$. Phosphorus is doped into Poly0 from SOG by this anneal. On the planarized surface, $2.0 \mu \mathrm{m}$ sputtered oxide is deposited by RF magnetron sputter. In order to improve the uniformity of the oxide thickness, the wafer is rotated during the sputtering. On the sputtered oxide, SOG is coated again and annealed for doping Poly1 with phosphorus. (e) The wafer is then patterned with the mask of dimples and etched by RIE. The depth of dimples is $0.75 \mu \mathrm{m}$. The roles of the dimples are (1) prevention of sticking between the beam and substrate, and (2) reinforcement of the non-stressed region by a kind of rib structure. After dimple etch, a next mask for anchor is applied and etched by RIE. (f) Then, the structural layer of polysilicon (Poly1) is deposited with a thickness of $2 \mu \mathrm{m}$ by LPCVD. (g) Again, the SOG is coated over the Polyl and the wafer is annealed at $1100^{\circ} \mathrm{C}$ for $90 \mathrm{~min}$. This anneal is not only for doping of the phosphorus from the top and bottom but also for reducing internal stress of Poly1. (h) The

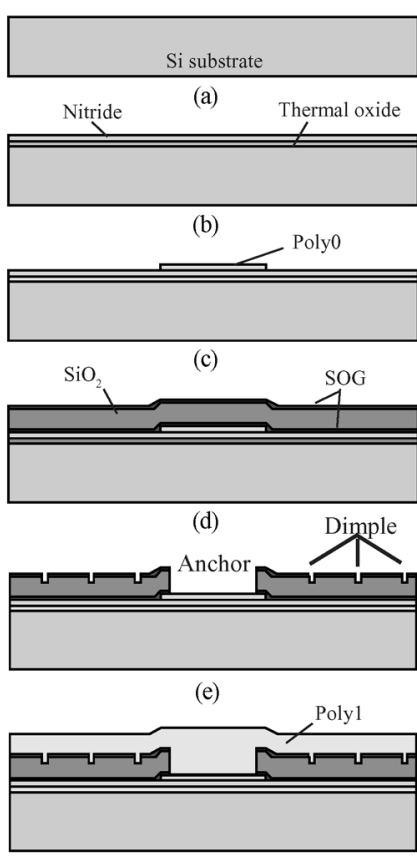

(f)

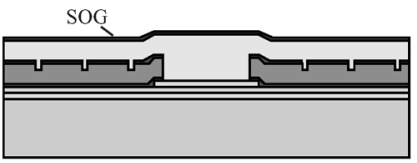

(g)

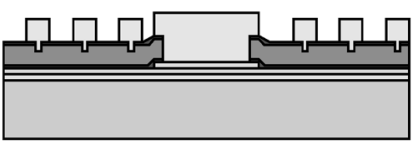

(h)

(i)

(j)
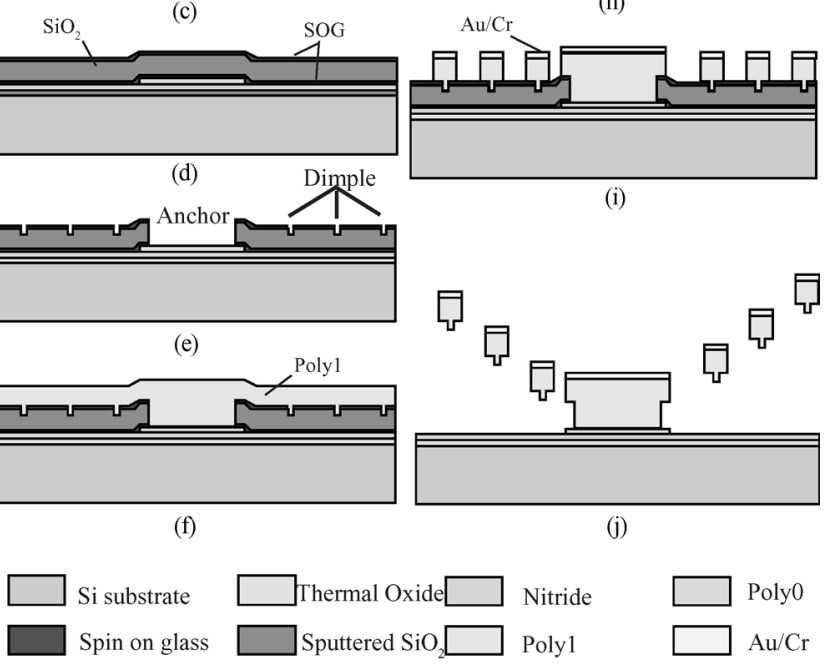

Fig. 11. Fabrication process

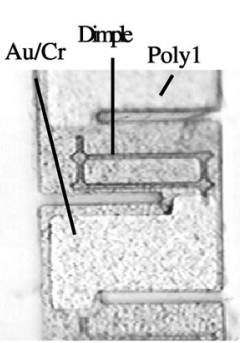

(a) One cell

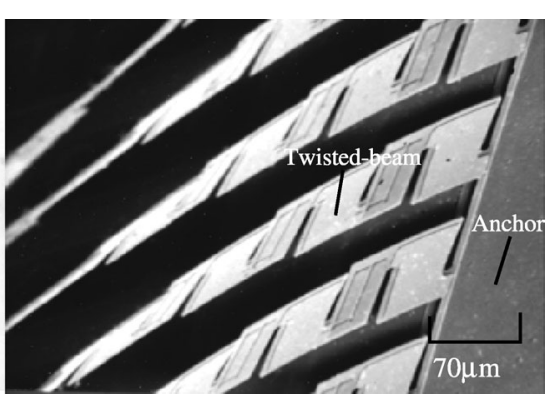

(b) Straight beams with different number of cells
Fig. 12. Photograph of fabricated twisted-beams

Poly1 is patterned and etched using the SOG as a hard mask of the RIE. After the etching of Poly1 is completed, the SOG hard mask is removed using BHF. (i) Then, the bonding pads and the stresslayer are patterned and gold with a thin chromium layer is deposited by a electron-beam evaporator. By lift-off of the metals, the final device with a sacrificial layer is obtained. (j) Finally, the structural layer of Poly1 is released by immersing the chips in a bath of $49 \% \mathrm{HF}$ for $15 \mathrm{~min}$. The chip is rinsed in deionized water, isopropyl alcohol and tertiary butyl alcohol, in order. The chip is then freeze-dried. Fig. 12 shows the photograph of the fabricated twisted-beams.

\section{Experiments}

At first, we estimated the internal stress of the $\mathrm{Au} / \mathrm{Cr}$ layer by measuring the curvature of the test wafer with the $\mathrm{Au} / \mathrm{Cr}$ layer ${ }^{(13)}$. The stress was estimated to be around $200 \mathrm{MPa}$ which is consistent with the designed value as described in Section 3.

As clearly shown in Fig. 12, the test device of the twisted-beam shows both curved-angle and twisted-angle. By measuring microscope, we examined the curved- and twisted-angles for 


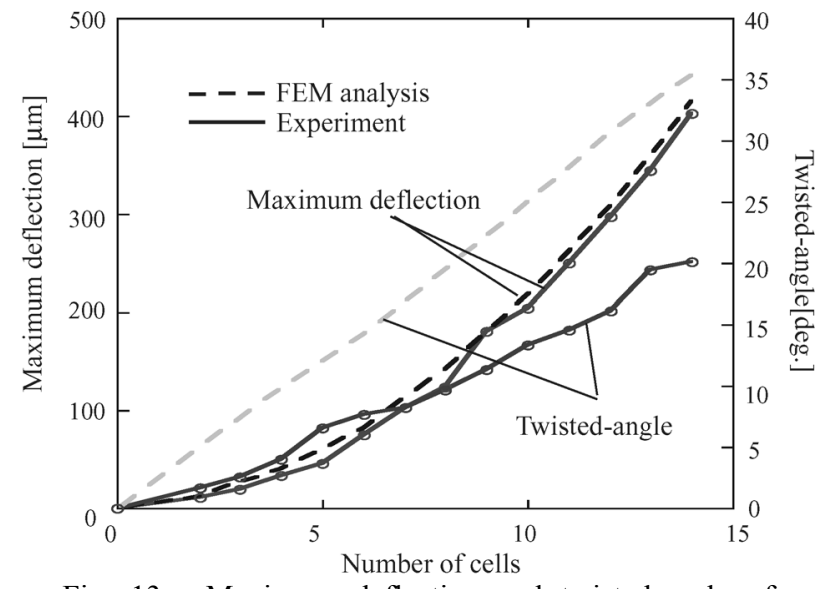

Fig. 13. Maximum deflection and twisted-angle of twisted-beams(Straight beam)

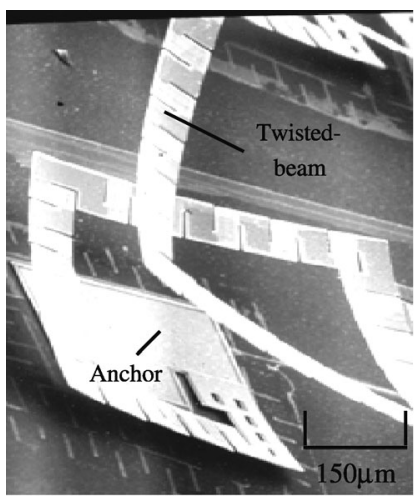

(a) With twisted-beams

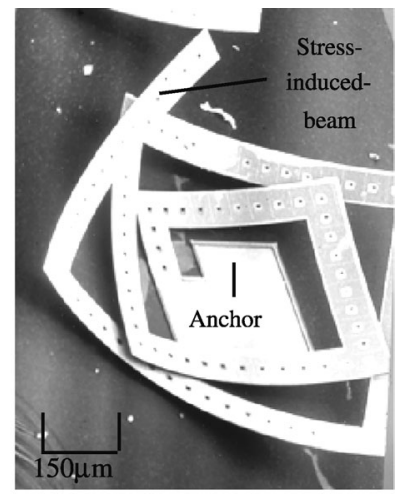

(b) With conventional-beams
Fig. 14. SEM photographs of fabricated electrostatic spiral actuator

Table 1. Displacement at each position of the spiral actuator

\begin{tabular}{|c|c|c|c|}
\hline & X-axis $[\mu \mathrm{m}]$ & Y-axis $[\mu \mathrm{m}]$ & Z-axis $[\mu \mathrm{m}]$ \\
\hline (a) & 0 & 2 & 16.4 \\
\hline (b) & 13 & 13 & 74.6 \\
\hline (c) & 39 & -1 & 140 \\
\hline (d) & 29 & -65 & 213 \\
\hline (e) & -54 & -48 & 253 \\
\hline (f) & -16 & 104 & 411 \\
\hline (g) & 156 & 61 & 481 \\
\hline (h) & 93 & -187 & 612 \\
\hline (i) & -263 & -108 & 749 \\
\hline
\end{tabular}

straight beams with a number of cells as a parameter. The measured values are shown in Fig. 13 with FEM analysis results. The measured maximum deflection or curved-angle agrees well with the analysis, but there are some differences for the twisted-angles. This difference may be due to miss alignment of the dimple pattern. In the design, the dimple is arranged at center of the non-stressed region to reinforce it. However, in the actual device, the dimple pattern was shifted towered the stress-induced region (cf. Fig. 12(a)) resulting in the reduced curvature of the stress-induced region at the off-centered link. Thus the measured twisted-angle becomes smaller than the results from FEM analysis.

Fig. 14 shows SEM photographs of the spiral actuators with twisted-beams (a) and conventional stress-induced beams (b). Clearly, large deflection can be seen in the actuator with

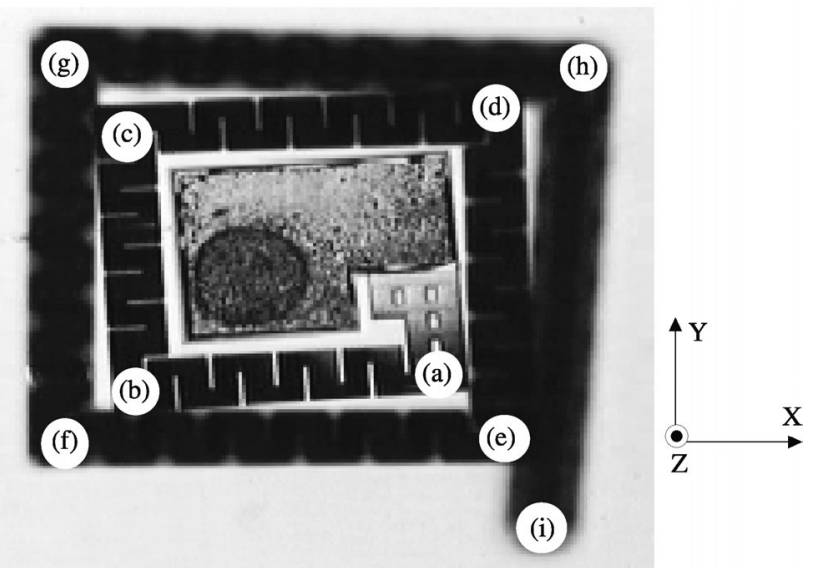

Fig. 15. Top view of the spiral actuator

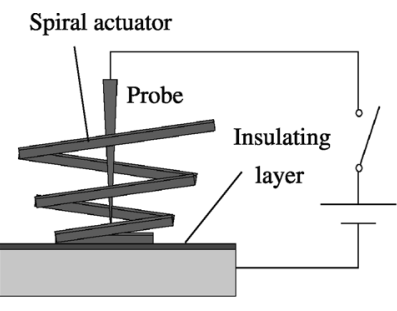

(a) No voltage applied

Fig. 16. Driving method of electrostatic spiral actuator

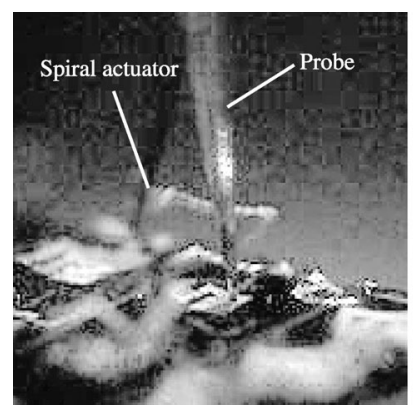

(a) No voltage applied

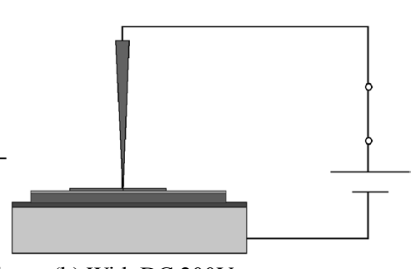

(b) With DC 200V
Fig. 17. Operation of electrostatic spiral actuator

twisted-beams. On the contrary, the actuator with conventional beams not only has small deflection but also becomes entangled. The detailed discussion is omitted here, but this phenomenon, the torsional deviation in $\mathrm{x}-\mathrm{y}$ plane, can be theoretically explained for the actuators with relatively large stress in the stress-induced region. Table 1 shows the measured displacement of the corners (points (a)-(i) in Fig. 15) of the spiral. Maximum displacement to $\mathrm{z}$-axis of $750 \mu \mathrm{m}$ was obtained, whereas the analytical value was $930 \mu \mathrm{m}$. This difference is results from individual effect of the miss-alignment of the dimple patterns for each beam. The displacement or shift in $\mathrm{x}-\mathrm{y}$ plane was also observed, however this shift is much smaller than the conventional one (Fig. 14). This characteristic must be taken into account for the system design including a load of the actuators.

The operation of the actuator was confirmed by applying DC voltage between the spiral and the substrate as shown in Fig. 16. The voltage of $200 \mathrm{~V}$ generates enough force to pull down whole spiral structure to the substrate. The photographs in Fig. 17 show the perspective views of the structure with and without driving voltage. Complete stroke was obtained with maximum response time of around $0.5 \mathrm{~s}$. 


\section{Conclusions}

The novel electrostatic stress-induced actuator with large stroke was described. The actuator is composed of "twisted-beams" which have out-of-plane curvature and rotation along its longitudinal direction. By using twisted-beams for spiral actuator, the total stroke becomes a sum of each deflection of the beams, contrary to conventional stress-induced actuators. The example device of $800 \times 800 \mu \mathrm{m}^{2}$ shows maximum stroke of $750 \mu \mathrm{m}$, which is extremely large stroke in stress-induced actuators. This actuator is useful for an large deflection optical mirror or anywhere the large displacement is required. In current stage, generation force is somewhat small, however, it can be improved by redesigning the cell of twisted-beam (e.g. larger cell) for individual applications.

(Manuscript received Aug. 29, 2005, revised Feb. 3, 2006)

\section{References}

(1) T. Yasuda and G. Miyamoto : "Low Voltage Electrostatic Microactuator with Large Deflection", Proc. of the 19th Sensor Symposium, pp.233-236 (2002)

(2) J. Li, M. P. Brenner, J. H. Lang, A. H. Slocum, and R. Struempler : "DRIE-FABRICATED CURVED-ELECTRODE ZIPPING ACTUATORS WITH LOW VOLTAGE", Dig. of Tech. Papers on The 12th Int. Conf. on Solid-State Sensors and Actuators (Transducers'03), Boston, USA, pp.480-483 (2003)

(3) S. H. Kim, K. Najafi, P. D. Washabaugh, and L. P. Bernal : "LARGE-DEFLECTION OUT-OF-PLANE ELECTROSTATIC BUCKLED-ELECTRODE ACTUATORS", Dig. of Tech. Papers on The 12th Int. Conf. on Solid-State Sensorsand Actuators (Transducers'03), Boston, USA, pp.794-797 (2003)

(4) R. Legtenberg, J. Gilbert, and S. D. Senturia : "Electrostatic Curved Electrode Actuators," J. of Microelectromech. Syst., Vol.6, No.3, pp.257-265 (1997)

(5) C. Chang and P. Chang : "INNOVATIVE MICROMACHINED MICROWAVE SWITCH WITH VERY LOW INSERTION LOSS", Dig. of Tech. Papers on The 10th Int. Conf. on Solid-State Sensors and Actuators (Transducers'99), Sendai, JAPAN, pp.1830-1833 (1999)

(6) Y. Takagi, S. Nakamichi, T. Fujita, K. Maenaka, and Y. Takayama : "Large displacement switch with curved beam for RF-MEMS", Proc. of the 2004 IEICE General Conference, p.9 (2004) (in Japanese).

(7) J. H. Babaei, C. Y. Kwok, and R. S. Huang : "INTEGLABLE ACTIVE MICROVALVE WITH SURFACE MICROMACHINED CURLEDUP ACTUATOR", Dig. of Tech. Papers on The 9th Int. Conf. on Solid-State Sensors and Actuators (Transducers'97), Chicago, USA, pp.833-836 (1997)

(8) C. L. Tsai and A. K. Henning : "Surface Micromachining Turbines", Dig. of Tech. Papers on The 9th Int. Conf. on Solid-State Sensors and Actuators (Transducers'97), Chicago, USA, pp.829-832 (1997)

(9) R. T. Chen, H. Nguyen, and M. C. Wu (UCLA) : "A LOW VOLTAGE MICROMACHINED OPTICAL SWITCH BYSTRESSINDUCED BENDING", MEMS2000, pp.424-428 (1999)

(10) Y. Kawamoto, T. Matsuda, and T. Yasuda : "Electrostatic Microactuator with Au/Poly-Si Bimorph Structure", J. of Microelectromech. Syst., Vol.14, No.2, pp.243-253 (2005)

(11) T. Fukushige, S. Hata, and A. Shimokohbe : "A MEMS Conical Spring Actuator Array", Proc. of the 21st Sensor Symposium, pp.43-46 (2004)

(12) S. B. Felch and J. S. Sonico : Varian Research Lab. Solid State Technology (1986)

(13) G. G. Stoney, Proc. R. Soc. London Ser A 82, 172 (1909)

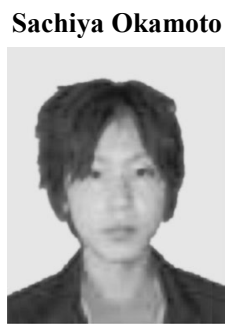

(Student Member) was born in Hyogo, Japan, in 1982. He received the B. E. degree from Himeji Institute of Technology, Japan, in 2004. He is currently working towards $M$. E. degree in electronics at University of Hyogo. His research topics are Poly-Si surface micromachining and technology of silicon micromachines.

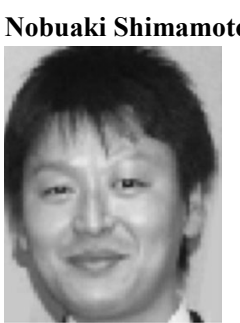

(Non-Member) was born in Hyogo, Japan, in 1980. $\mathrm{He}$ received the B. E. and M. E. degrees from Himeji Institute of Technology, Japan, in 2003 and 2005, respectively. His research topics were Poly-Si surface micromachining and technology of silicon icromachines.

Yuko Matsushita

(Non-Member) was born in Hyogo, Japan, in 1983. She received the B. E. degree from Himeji Institute of Technology, Japan, in 2005. Her research topics were silicon micromachined gyroscope and technology of silicon micromachines.

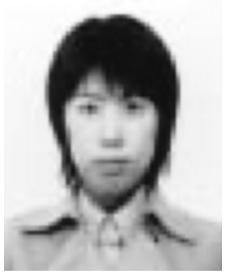

Takayuki Fujita

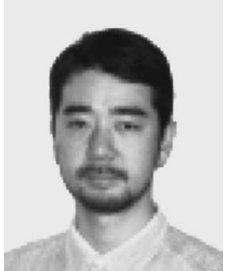

(Member) born in Hyogo, Japan, in 1972. He received the B. E., M. E. and $\mathrm{Ph}$. D. degrees from Himeji Institute of Technology, Japan, in 1995, 1997 and 2000, respectively. Since 2001, he has been with the Department of Electronics of Himeji Institute of Technology, where he is presently a research associate. By unification of universities in the Hyogo prefecture at April 2004, now he joined the University of Hyogo. His research topics are silicon MEMS and Integrated Circuit devices.

Kazusuke Maenaka (Member) was born in Hyogo, Japan, in 1959. He

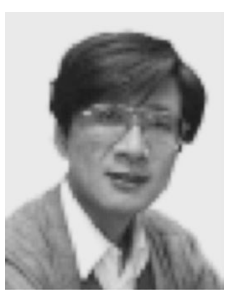
received the B. E., M. E. and $\mathrm{Ph}$. D. degrees from Toyohashi University of Technology, Japan, in 1982, 1984 and 1990, respectively. Since 1993, he has been with the Department of Electronics of Himeji Institute of Technology, where he is presently an associate professor. By unification of universities in the Hyogo prefecture at April 2004, now he joined the University of Hyogo. His research interests include silicon magnetic sensors and their integration, silicon mechanical sensors and actuators.

Yoichiro Takayama (Non-Member) was born in 1942, Japan, in Osaka.

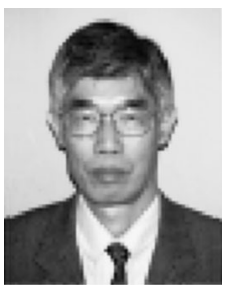
He received the B. E., M. E. and $\mathrm{Ph}$. D. degrees from Osaka University, Japan, in 1965, 1967 and 1973, respectively. From 1967 to 2000, he worked at NEC Corporation, where he was engaged in the research and development of microwave semiconductor devices and their circuit technologies. In 2001, he joined Himeji Institute of Technology as a professor and is currently engaged in the research and development of microwave devices, their circuit applications and MEMS technology. By unification of universities in the Hyogo prefecture at April 2004, now he joined the University of Hyogo. He received the 1983 Microwave Prize granted by IEEE MTT Society. 